\title{
A Subspace Multiuser Beamforming Algorithm for the Downlink in Mobile Communications
}

\author{
Nanyan Wang, Panajotis Agathoklis, and Andreas Antoniou
}

\begin{abstract}
A new algorithm for downlink multiuser beamforming in mobile communications is described. The optimization problem is reformulated by modifying the constraints so that weight vectors of different mobile stations are optimized in a reduced feasible region which is a subset of that of the multiuser beamforming problem. The downlink beamforming weight vectors of different mobile stations are then jointly optimized in a subspace instead of searching in the entire parameter space. Simulation results show that the modified optimization problem leads to solutions that satisfy the signal-to-noise-plus-interference ratio specification at each mobile station and that the total power transmitted from the base station is very close to the optimal one. The solution of the modified optimization problem requires significantly less computation than the optimal multiuser beamforming algorithms.
\end{abstract}

Index Terms-Antenna arrays, beamforming, downlink.

\section{INTRODUCTION}

C UURENT mobile communication systems suffer from multiple-access interference, which not only limits the system capacity but also significantly increases the required transmission power for quality service. One of the most promising approaches to meet these challenges is through the use of adaptive beamforming and power control [1], [2].

In the downlink of mobile communication systems, the goal of beamforming is to concentrate the power transmitted from the base station (BS) in the direction of the target mobile station (MS) and reduce interference on the downlink signals to other MSs. In the single-user beamforming (SUB) approach [3]-[5], the downlink weight vectors for different MSs are obtained by maximizing the downlink signal power to the target MS relative to the total power radiated in the direction of other MSs. Based on the weight vectors obtained, the BS transmitted power of different mobiles can then be computed to minimize the total BS transmitted power and satisfy the signal-to-noise-plus-interference ratio (SINR) specification at each MS [6]. The SUB approach is computationally simple but provides a suboptimal solution to the problem of minimizing the BS transmitted power.

In the multiuser beamforming (MUB) approach, the BS weight vectors for all MSs are jointly optimized. In [7], MUB is formulated as an optimization problem where the BS weight

Manuscript received April 9, 2004; revised April 28, 2005. This work was supported by the Natural Sciences and Engineering Research Council of Canada, Micronet, and the NCE Program. This paper was recommended by Associate Editor A. C. Kot.

The authors are with the Department of Electrical and Computer Engineering, University of Victoria, Victoria, BC V8W 3P6, Canada (e-mail: nwang@ece. uvic.ca; pan@ece.uvic.ca; aantoniou@ieee.org).

Digital Object Identifier 10.1109/TCSII.2006.875381 vectors for different MSs are jointly optimized so as to satisfy given SINR specifications at MSs and, at the same time, the total power transmitted from the base station (BS) is minimized. The optimal MUB turns out to be an optimization problem with nonconvex quadratic constraints. Two classes of algorithms have been developed for MUB, namely, duality-based [6], [10] and semidefinte programming (SDP)-based [11]-[13] MUB algorithms. The duality between the uplink and downlink was originally presented and discussed in [6]. It has been shown that the optimal downlink weight vectors can be obtained through the use of a virtual uplink. Based on duality, an optimal MUB algorithm for multicell is developed to iteratively obtain the optimal downlink weight vectors. An early version of the duality-based algorithm [6] tends to converge more slowly as the SINR requirements become more stringent. The duality between the uplink and downlink was further discussed in [8] and [9], and a new duality-based MUB algorithm was proposed in [10] where several stopping criteria are proposed to improve the convergence behavior of the iterative algorithm. An SDP-based MUB algorithm can be found in [11]-[13], where the optimal MUB optimization problem is relaxed into a SDP optimization problem after Lagrangian relaxation [14] The weight vectors are then obtained from the optimal solution of the SDP problem. However, the amount of computation required in this approach is high and it increases rapidly as the number of antenna elements is increased.

In this paper, a new downlink MUB technique for mobile communications is developed. An earlier version of this technique was presented in [16]. The MUB optimization problem in [11] is reformulated by modifying the SINR constraints so as to obtain a feasible region for the solution which is a subset of the region of the original optimization problem. The BS weight vectors of different MSs are then jointly optimized in their corresponding subspaces instead of the entire parameter space. Simulations of CDMA systems with various numbers of antenna elements show that the modified optimization problem leads to a solution that satisfies the SINR specification at each MS while the total power transmitted from the BS is very close to the optimal one. In addition, the solution requires significantly less computation than the optimal MUB.

\section{SySTEM MODEL}

Consider the MUB system illustrated in Fig. 1, where a $p$-element antenna array is deployed at the BS and an omnidirectional antenna with unit gain is deployed at each MS. Signal $c_{k}$ to MS $k$ is first split into $p$ signals corresponding to $p$ antenna elements, which are then weighted by the beamforming weights. The beamforming weights corresponding to different MSs, which determine the BS radiation pattern and downlink 


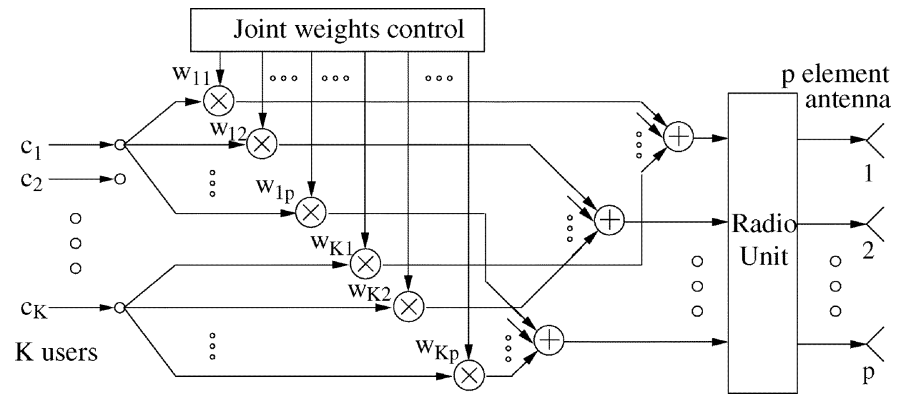

Fig. 1. Block diagram of a downlink beamforming system at the BS.

signal power, are jointly computed based on the channel information obtained. Then, the weighted signal components to different MSs are combined branch by branch and transmitted from each antenna element.

After down-converting to baseband, the desired downlink signal $x_{k, l}^{s}(t)$ received from the $l$ th path at MS $k$ is given by

$$
x_{k, l}^{s}(t)=\beta_{k, l} c_{k}\left(t-\tau_{k, l}\right) \mathbf{a}^{H}\left(\theta_{k, l}\right) \mathbf{w}_{k}
$$

where $\beta_{k, l}$ is the complex channel response for the $l$ th path of MS $k, c_{k}(t)$ is the normalized transmitted signal to MS $k, \tau_{k, l}$ is the path delay, $\mathbf{w}_{k}$ is the BS beamforming weight vector of MS $k, \theta_{k, l}$ is the direction of departure of the $l$ th path from BS antenna array to MS $k$, and $\mathbf{a}^{H}\left(\theta_{k, l}\right)$ is the $p$-dimension column vector known as the BS antenna-array response vector.

The cochannel interference $(\mathrm{CCI}) x_{k, l}^{I_{i}}(t)$ received through the $l$ th path at the target MS $k$ caused by the downlink signal to MS $i$ can be represented by

$$
x_{k, l}^{I_{i}}(t)=\beta_{k, l} c_{i}\left(t-\tau_{k, l}\right) \mathbf{a}^{H}\left(\theta_{k, l}\right) \mathbf{w}_{i} .
$$

The received signal at MS $k$ consists of the desired downlink signal and the CCI caused by the downlink signals to other MSs. If the number of MSs in a BS service area is $K$ and the number of dominant paths from the BS to MS $k$ is $L_{k}$, then the received signal at MS $k$ can be represented by

$$
x_{k}(t)=\sum_{l=1}^{L_{k}} x_{k, l}^{s}(t)+\sum_{\substack{i=1 \\ i \neq k}}^{K} \sum_{l=1}^{L_{k}} x_{k, l}^{I_{i}}(t)+n_{k}(t)
$$

where $n_{k}(t)$ is the noise which is assumed to have zero mean and covariance $\sigma_{k}^{2}$.

\section{DOWNLINK BEAMFORMING AND POWER CONTROL}

\section{A. Optimal Downlink MUB}

The optimal beamforming weights can be determined by minimizing the total power transmitted from the BS such that a given SINR specification is achieved. The power transmitted from the BS for each MS is proportional to the $L_{2}$-norm of the corresponding weight vector. Let $p_{k}^{t}$ denote the $L_{2}$-norm $\left\|\mathbf{w}_{k}\right\|^{2}$ of the BS weight vector $\mathbf{w}_{k}$ for mobile $k$. The downlink MUB optimization problem can be formulated as

$$
\begin{aligned}
\min & {\left[\sum_{k=1}^{K} p_{k}^{t}\right] } \\
\text { subject to } & f_{k}\left(\mathbf{w}_{1}, \mathbf{w}_{2}, \ldots, \mathbf{w}_{K}\right) \geq \gamma_{k}, \\
\text { for } & k=1,2, \ldots, K
\end{aligned}
$$

where

$$
f_{k}\left(\mathbf{w}_{1}, \mathbf{w}_{2}, \ldots, \mathbf{w}_{K}\right)=\frac{p_{k, k}^{r}}{\sum_{\substack{j=1 \\ j \neq i}}^{K} p_{k, j}^{r}+\sigma_{k}^{2}} .
$$

In the above equation, $\sigma_{k}$ is the noise variance at MS $k, \gamma_{k}$ is the required minimum SINR at MS $k$,

$$
p_{k, k}^{r}=\sum_{l=1}^{L_{k}} E\left|x_{k, l}^{s}\right|^{2}=\mathbf{w}_{k}^{H} \mathbf{R}_{k} \mathbf{w}_{k}
$$

is the received downlink signal power at MS $k$, and

$$
p_{k, j}^{r}=\sum_{l=1}^{L_{k}} E\left|x_{k, l}^{I_{j}}\right|^{2}=\mathbf{w}_{j}^{H} \mathbf{R}_{k} \mathbf{w}_{j}
$$

is the received power of the CCI at MS $k$ caused by the downlink signal to MS $j$. Matrix $\mathbf{R}_{k}$ of the downlink signal to MS $k$ is given by

$$
\mathbf{R}_{k}=\sum_{l=1}^{L_{k}} E\left[\left|\beta_{k, l}\right|^{2}\right] \mathbf{a}\left(\theta_{k, l}\right) \mathbf{a}^{H}\left(\theta_{k, l}\right) .
$$

Matrix $\mathbf{R}_{k}$, for $k=1,2, \ldots, K$, needs to be estimated in order to solve the MUB problem. In the case of time-division duplex systems where the uplink and downlink channels are reciprocal, it can be obtained through uplink channel estimation [6]. In the case of frequency-division duplex systems where the frequency channels used in the downlink and uplink are different, matrix $\mathbf{R}_{k}$, for $k=1,2, \ldots, K$, can be estimated via feedback signaling [10], [15].

\section{B. New Downlink MUB Algorithm}

In this section, a new MUB approach is presented. The basic idea is to optimize the BS weights for each MS in a subspace spanned by its antenna response vectors and, therefore, reduce the number of variables considered in the SDP problem. As will be shown, the proposed algorithm requires less computation than the optimal MUB while maintaining the total power transmitted from the BS at a level close to the optimal one.

The proposed approach is based on the decomposition of the range space of $\mathbf{R}_{k}$. Matrix $\mathbf{R}_{k}$ in (8) is Hermitian and positive semidefinite and, hence, it has real and nonnegative eigenvalues. It can be decomposed as

$$
\mathbf{R}_{k}=\mathbf{U}_{k} \Lambda_{k} \mathbf{U}_{k}^{H}
$$

where $\Lambda_{k}=\operatorname{diag}\left\{\lambda_{1}, \lambda_{2}, \ldots, \lambda_{p}\right\}$ and $\mathbf{U}_{k}=\left[\mathbf{v}_{k}^{1}, \mathbf{v}_{k}^{2}, \ldots, \mathbf{v}_{k}^{p}\right]$. Since $\left\{\mathbf{v}_{k}^{1}, \mathbf{v}_{k}^{2}, \ldots, \mathbf{v}_{k}^{p}\right\}$ is a set of orthonormal eigenvectors of 
matrix $\mathbf{R}_{k}$ which is a basis of vector space $C$, the weight vector $\mathbf{w}_{k}$ can be expressed as

$$
\mathbf{w}_{k}=\mathbf{U}_{k} \mathbf{x}_{k} .
$$

Generally, the rank of matrix $\mathbf{R}_{k}$ is less than $p$. In such a case, the span of $\left\{\mathbf{v}_{k}^{1}, \mathbf{v}_{k}^{2}, \ldots, \mathbf{v}_{k}^{s_{k}}\right\}$, which is the set of the eigenvectors associated with the $s_{k}$ nonzero eigenvalues $\lambda_{1}, \lambda_{2}, \ldots, \lambda_{s_{k}}$, is the subspace $\hat{C}_{k}$ of the downlink signal to MS $k$. The subspace $\hat{C}_{k}$ can also be selected as the set of the eigenvectors associated with the largest $s_{k}$ eigenvalues due to the presence of noise component. The span of the remaining eigenvectors $\mathbf{v}_{k}^{s_{k}+1}, \mathbf{v}_{k}^{s_{k}+2}, \ldots, \mathbf{v}_{k}^{p}$ is the orthogonal complement $\bar{C}_{k}$ of the subspace $\hat{C}_{k}$. If we let

$$
\begin{aligned}
\hat{\mathbf{U}}_{k} & =\left[\begin{array}{llll}
\mathbf{v}_{k}^{1} & \mathbf{v}_{k}^{2} & \cdots & \mathbf{v}_{k}^{s_{k}}
\end{array}\right] \\
\overline{\mathbf{U}}_{k} & =\left[\begin{array}{llll}
\mathbf{v}_{k}^{s_{k}+1} & \mathbf{v}_{k}^{s_{k}+2} & \cdots & \mathbf{v}_{k}^{p}
\end{array}\right]
\end{aligned}
$$

then matrix $\mathbf{R}_{k}$ can be rewritten as

$$
\mathbf{R}_{k}=\hat{\mathbf{U}}_{k} \hat{\Lambda}_{k} \hat{\mathbf{U}}_{k}^{H}
$$

where $\hat{\Lambda}_{k}=\operatorname{diag}\left\{\lambda_{1}, \lambda_{2}, \ldots, \lambda_{s_{k}}\right\}$. Vector $\mathbf{x}_{k}$ can now be partitioned as

$$
\mathbf{x}_{k}=\left[\begin{array}{l}
\hat{\mathbf{x}}_{k} \\
\overline{\mathbf{x}}_{k}
\end{array}\right]
$$

where the $s_{k}$ components of vector $\hat{\mathbf{x}}_{k}$ and the $p-s_{k}$ components of vector $\overline{\mathbf{x}}_{k}$ are the components of $\mathbf{x}_{k}$ in subspace $\hat{C}_{k}$ and its orthogonal complement $\bar{C}_{k}$, respectively. Thus, the weight vector $\mathbf{w}_{k}$ can be rewritten as

$$
\mathbf{w}_{k}=\left[\begin{array}{ll}
\hat{\mathbf{U}}_{k} & \overline{\mathbf{U}}_{k}
\end{array}\right]\left[\begin{array}{l}
\hat{\mathbf{x}}_{k} \\
\overline{\mathbf{x}}_{k}
\end{array}\right]=\hat{\mathbf{U}}_{k} \hat{\mathbf{x}}_{k}+\overline{\mathbf{U}}_{k} \overline{\mathbf{x}}_{k} .
$$

Since

$$
\begin{aligned}
\hat{\mathbf{U}}_{k}^{H} \hat{\mathbf{U}}_{k} & =\mathbf{I}_{s_{k}} \\
\overline{\mathbf{U}}_{k}^{H} \overline{\mathbf{U}}_{k} & =\mathbf{I}_{p-s_{k}} \\
\hat{\mathbf{U}}_{k}^{H} \overline{\mathbf{U}}_{k} & =0
\end{aligned}
$$

the BS transmiited poser $p_{k}^{t}$ for MS $k$ can be derived as

$$
p_{k}^{t}=\left\|\mathbf{w}_{k}\right\|^{2}=\left\|\hat{\mathbf{U}}_{k} \hat{\mathbf{x}}_{k}+\overline{\mathbf{U}}_{k} \overline{\mathbf{x}}_{k}\right\|^{2}=\hat{p}_{k}^{t}+\bar{p}_{k}^{t}
$$

where $\hat{p}_{k}^{t}=\left\|\hat{\mathbf{x}}_{k}\right\|^{2}$ and $\bar{p}_{k}^{t}=\left\|\overline{\mathbf{x}}_{k}\right\|^{2}$ are the power in the subspace $\hat{C}_{k}$ and its orthogonal complement $\bar{C}_{k}$, respectively. If we substitute (13) into (6), the received signal power $p_{k, k}^{r}$ at MS $k$ can be expressed as

$$
\begin{aligned}
p_{k, k}^{r} & =\left[\begin{array}{ll}
\hat{\mathbf{x}}_{k}^{H} & \overline{\mathbf{x}}_{k}^{H}
\end{array}\right]\left[\begin{array}{l}
\hat{\mathbf{U}}_{k}^{H} \\
\overline{\mathbf{U}}_{k}^{H}
\end{array}\right] \hat{\mathbf{U}}_{k} \hat{\Lambda}_{k} \hat{\mathbf{U}}_{k}^{H}\left[\begin{array}{cc}
\hat{\mathbf{U}}_{k} & \overline{\mathbf{U}}_{k}
\end{array}\right]\left[\begin{array}{c}
\hat{\mathbf{x}}_{k} \\
\overline{\mathbf{x}}_{k}
\end{array}\right] \\
& =\hat{\mathbf{x}}_{k}^{H} \hat{\mathbf{U}}_{k}^{H} \hat{\mathbf{U}}_{k} \hat{\Lambda}_{k} \hat{\mathbf{U}}_{k}^{H} \hat{\mathbf{U}}_{k} \hat{\mathbf{x}}_{k} .
\end{aligned}
$$

If we define $\mathbf{R}_{k}^{1 / 2}=\hat{\Lambda}_{k}^{1 / 2} \hat{\mathbf{U}}_{k}^{H}$ where $\hat{\Lambda}_{k}^{1 / 2}=$ $\operatorname{diag}\left\{\lambda_{1}^{1 / 2}, \lambda_{2}^{1 / 2}, \ldots, \lambda_{s_{k}}^{1 / 2}\right\}$, the received signal power $p_{k, k}^{r}$ in (18) can be rewritten as

$$
p_{k, k}^{r}=\left\|\mathbf{R}_{k}^{\frac{1}{2}} \hat{\mathbf{U}}_{k} \hat{\mathbf{x}}_{k}\right\|^{2}
$$

Similarly, the received power of CCI $p_{k, j}^{r}$ at MS $k$ caused by downlink signal to MS $j$ can be expressed as

$$
p_{k, j}^{r}=\left\|\mathbf{R}_{k}^{\frac{1}{2}} \hat{\mathbf{U}}_{j} \hat{\mathbf{x}}_{j}+\mathbf{R}_{k}^{\frac{1}{2}} \overline{\mathbf{U}}_{j} \overline{\mathbf{x}}_{j}\right\|^{2} .
$$

Applying the triangle inequality to (20), we have

$$
p_{k, j}^{r} \leq \hat{p}_{k, j}^{r}+\bar{p}_{k, j}^{r}
$$

where

$$
\begin{aligned}
& \hat{p}_{k, j}^{r}=\left\|\mathbf{R}_{k}^{\frac{1}{2}} \hat{\mathbf{U}}_{j} \hat{\mathbf{x}}_{j}\right\|^{2} \\
& \bar{p}_{k, j}^{r}=\left\|\mathbf{R}_{k}^{\frac{1}{2}} \overline{\mathbf{U}}_{j} \overline{\mathbf{x}}_{j}\right\|^{2}+2\left\|\mathbf{R}_{k}^{\frac{1}{2}} \hat{\mathbf{U}}_{j} \hat{\mathbf{x}}_{j}\right\|\left\|\mathbf{R}_{k}^{\frac{1}{2}} \overline{\mathbf{U}}_{j} \overline{\mathbf{x}}_{j}\right\| .
\end{aligned}
$$

Let us now consider a modified optimization problem that has the same objective function as the optimization problem in (4) but different constraints from those in (5), i.e.,

$$
\begin{aligned}
\min & {\left[\sum_{k=1}^{K}\left(\hat{p}_{k}^{t}+\bar{p}_{k}^{t}\right)\right] } \\
\text { subject to } & g_{k}\left(\mathbf{x}_{1}, \mathbf{x}_{2}, \ldots, \mathbf{x}_{K}\right) \geq \gamma_{k}, \\
\text { for } & k=1,2, \ldots, K
\end{aligned}
$$

where

$$
g_{k}\left(\mathbf{x}_{1}, \mathbf{x}_{2}, \ldots, \mathbf{x}_{K}\right)=\frac{p_{k, k}^{r}}{\sum_{\substack{j=1 \\ j \neq i}}^{K}\left(\hat{p}_{k, j}^{r}+\bar{p}_{k, j}^{r}\right)+\sigma_{k}^{2}} .
$$

Proposition 1: The feasible solution of the modified optimization problem in (24) satisfies the SINR specification at each MS.

Proof: Let $\Re^{f}$ and $\Re^{g}$ denote the feasible regions of the original optimization problems in (4), namely, (5), and the modified optimization problem in (24), namely, (25), respectively. Consider a feasible solution $\left(\mathbf{x}_{1}, \mathbf{x}_{2}, \ldots, \mathbf{x}_{K}\right)$ in $\Re^{g}$ such that

$$
g_{k}\left(\mathbf{x}_{1}, \mathbf{x}_{2}, \ldots, \mathbf{x}_{K}\right) \geq \gamma_{k}, \quad \text { for } k=1,2, \ldots, K .
$$

From (21), it can be readily shown that

$$
\begin{aligned}
& f_{k}\left(\mathbf{x}_{1}, \mathbf{x}_{2}, \ldots, \mathbf{x}_{K}\right) \geq g_{k}\left(\mathbf{x}_{1}, \mathbf{x}_{2}, \ldots, \mathbf{x}_{K}\right), \\
& \text { for } k=1,2, \ldots, K .
\end{aligned}
$$

and hence it follows that

$$
f_{k}\left(\mathbf{x}_{1}, \mathbf{x}_{2}, \ldots, \mathbf{x}_{K}\right) \geq \gamma_{k}, \quad \text { for } k=1,2, \ldots, K \text {. }
$$

Therefore, $\Re^{g} \subseteq \Re^{f}$, and any feasible solution in $\Re^{g}$ is also a feasible solution in $\Re^{f}$. Thus, the modified optimization problem leads to a solution that satisfies the SINR specification at each MS.

Proposition 2: The optimal solution of the modified optimization problem in (24) is obtained when $\overline{\mathbf{x}}_{k}=0$ for $i=$ $1,2, \ldots, K$.

Proof: The proposition will be proved by contradiction. Assume that the optimal solution of the problem in (24) has the form

$$
\mathbf{w}_{k}^{A}=\hat{\mathbf{U}}_{k} \hat{\mathbf{x}}_{k}^{a}+\overline{\mathbf{U}}_{k} \overline{\mathbf{x}}_{k}^{a}, \quad \text { for } k=1,2, \ldots, K
$$

and $\left\|\overline{\mathbf{x}}_{k}^{a}\right\|^{2} \neq 0$ for at least one MS. Consider a nonoptimal solution

$$
\mathbf{w}_{k}^{B}=\hat{\mathbf{U}}_{k} \hat{\mathbf{x}}_{k}^{a}, \quad \text { for } k=1,2, \ldots, K .
$$


Quantities $g_{k}\left(\mathbf{x}_{1}^{A}, \mathbf{x}_{2}^{A}, \ldots, \mathbf{x}_{K}^{A}\right)$ and $g_{k}\left(\mathbf{x}_{1}^{B}, \mathbf{x}_{2}^{B}, \ldots, \mathbf{x}_{K}^{B}\right)$ for $k=1,2, \ldots, K$ can then be obtained by substituting (26) and (27) into (25), which leads to

$$
\begin{aligned}
g_{k}\left(\mathbf{x}_{1}^{B}, \mathbf{x}_{2}^{B}, \ldots, \mathbf{x}_{K}^{B}\right) & \geq g_{k}\left(\mathbf{x}_{1}^{A}, \mathbf{x}_{2}^{A}, \ldots, \mathbf{x}_{K}^{A}\right) \\
& \geq \gamma_{k}, \quad \text { for } k=1,2, \ldots, K .
\end{aligned}
$$

All of the constraints in (24) are satisfied using the weights $\mathbf{w}_{1}^{B}, \mathbf{w}_{2}^{B}, \ldots, \mathbf{w}_{K}^{B}$ and, therefore, $\mathbf{x}_{1}^{B}, \mathbf{x}_{2}^{B}, \ldots, \mathbf{x}_{K}^{B}$ is a set of feasible solutions. Substituting (26) and (27) into the objective function in (24), we obtain the total BS transmitted powers as

$$
\begin{aligned}
p^{t}\left(\mathbf{x}_{1}^{A}, \mathbf{x}_{2}^{A}, \cdots, \mathbf{x}_{K}^{A}\right) & =\sum_{k=1}^{K}\left(\hat{\mathbf{x}}_{k}^{H} \hat{\mathbf{x}}_{k}+\overline{\mathbf{x}}_{k}^{H} \overline{\mathbf{x}}_{k}\right) \\
p^{t}\left(\mathbf{x}_{1}^{B}, \mathbf{x}_{2}^{B}, \cdots, \mathbf{x}_{K}^{B}\right) & =\sum_{k=1}^{K} \hat{\mathbf{x}}_{k}^{H} \hat{\mathbf{x}}_{k}
\end{aligned}
$$

respectively. Hence, from (28) and (29), it follows that

$$
p^{t}\left(\mathbf{x}_{1}^{B}, \mathbf{x}_{2}^{B}, \ldots, \mathbf{x}_{K}^{B}\right)<p^{t}\left(\mathbf{x}_{1}^{A}, \mathbf{x}_{2}^{A}, \ldots, \mathbf{x}_{K}^{A}\right) .
$$

This leads to the contradiction that the nonoptimal beamforming weights $\mathbf{w}_{1}^{B}, \mathbf{w}_{2}^{B}, \ldots, \mathbf{w}_{K}^{B}$ are better than the optimal beamforming weights $\mathbf{w}_{1}^{A}, \mathbf{w}_{2}^{A}, \ldots, \mathbf{w}_{K}^{A}$. This contradiction implies that the optimal beamforming weight vectors of the modified optimization problem in (24) are obtained when $\overline{\mathbf{x}}_{k}=0$ for $k=1,2, \ldots, K$.

Since only the $s_{k}$ components of vector $\hat{\mathbf{x}}_{k}$, for $k=$ $1,2, \ldots, K$, need to be considered, the optimization problem in (24) can be reformulated as

$$
\begin{aligned}
& \min {\left[\sum_{k=1}^{K}\left\|\hat{\mathbf{x}}_{k}\right\|^{2}\right] } \\
& \text { subject to } \frac{\left\|\mathbf{R}_{k}^{\frac{1}{2}} \hat{\mathbf{U}}_{k} \hat{\mathbf{x}}_{k}\right\|^{2}}{\sum_{\substack{j=1 \\
j \neq k}}^{K}\left\|\mathbf{R}_{k}^{\frac{1}{2}} \hat{\mathbf{U}}_{j} \hat{\mathbf{x}}_{j}\right\|^{2}+\sigma_{k}^{2}} \geq \gamma_{k} \\
& \text { for } \quad k=1,2, \ldots, K .
\end{aligned}
$$

Further, if we define $\hat{\mathbf{X}}_{k}=\hat{\mathbf{x}}_{k} \hat{\mathbf{x}}_{k}^{H}$ for $k=1,2, \ldots, K$ and relax the constraint $\operatorname{Rank}\left(\hat{\mathbf{X}}_{k}\right)=1$ implied by this definition, then the optimization problem in (31) can be rewritten as an SDP problem of the form

$$
\begin{aligned}
\min _{\hat{\mathbf{X}}} & \sum_{k=1}^{K} \operatorname{Tr}\left[\hat{\mathbf{X}}_{k}\right] \\
\text { subject to } & \operatorname{Tr}\left[\hat{\mathbf{U}}_{k}^{H} \mathbf{R}_{k} \hat{\mathbf{U}}_{k} \hat{\mathbf{X}}_{k}\right] \\
& \quad-\gamma_{k} \sum_{\substack{j=1 \\
j \neq k}}^{K} \operatorname{Tr}\left[\hat{\mathbf{U}}_{j}^{H} \mathbf{R}_{k} \hat{\mathbf{U}}_{j} \hat{\mathbf{X}}_{j}\right]-\sigma_{k}^{2} \gamma_{k} \geq 0 \\
& \hat{\mathbf{X}}_{k}=\hat{\mathbf{X}}_{k}^{H} \\
& \hat{\mathbf{X}}_{k} \succeq 0 \\
\text { for } \quad & k=1,2, \ldots, K .
\end{aligned}
$$

Based on the solution $\hat{\mathbf{X}}_{k}$, for $k=1,2, \ldots, K$, of the optimization problem in (32), $\hat{\mathbf{x}}_{k}$ can be calculated as

$$
\hat{\mathbf{x}}_{k}=\sqrt{\mu_{k}} \mathbf{q}_{k}, \quad \text { for } k=1,2, \ldots, K
$$

TABLE I

Proposed Subspace MUB Algorithm

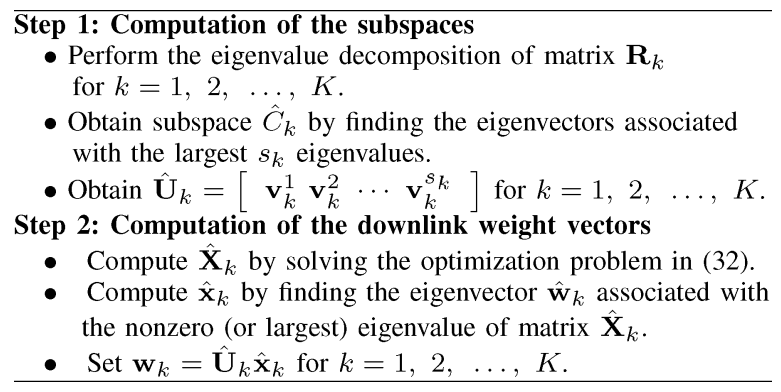

where $\mathbf{q}_{k}$ is the eigenvector associated with the nonzero (or largest) eigenvalue $\mu_{k}$ of matrix $\hat{\mathbf{X}}_{k}$. Hence, from (13), the weight vectors can be obtained as

$$
\mathbf{w}_{k}=\hat{\mathbf{U}}_{k} \hat{\mathbf{x}}_{k}, \quad \text { for } k=1,2, \ldots, K \text {. }
$$

An algorithm based on the method described is summarized in Table I.

The proposed MUB algorithm solves the modified optimization problem in (32) instead of the original optimization problem in (4). Because of proposition 1, the solution of the former will satisfy the SINR specifications of the original optimization problem. Furthermore, the solution can be obtained in a subspace of the optimization parameter space of the original problem due to proposition 2 . The number of variables to be considered in the modified optimization problem and the original optimization problem, $n^{s}$ and $n^{o}$, respectively, can be readily obtained as

$$
\begin{aligned}
n^{s} & =\sum_{k=1}^{K}\left(s_{k}+1\right) s_{k} / 2 \\
n^{o} & =K(p+1) p / 2 .
\end{aligned}
$$

The use of more antenna elements is an effective way to reduce the transmitted power, improve the quality of service, and increase the capacity of a mobile communication system. However, it can be shown from (36) that the number of variables required in the original optimization problem is of order $O\left(p^{2}\right)$ and thus its computational complexity increases rapidly with the number of antenna elements. This tends to render the problem less tractable for real-time applications. In contrast, the number of variables in the modified optimization problem depends only on the downlink channels. Since $s_{k} \leq p$ and, in most cases, $s_{k}<p$ for $k=1,2, \ldots, K, n^{s}$ is much smaller than $n^{o}$. Thus, the solution of the modified optimization problem requires significantly less computation than the optimal MUB. In addition, as will be illustrated in Section IV, the power transmitted by the BS is close to that of the optimal MUB. In the extreme case where $s_{k}=p$, for $k=1,2, \ldots, K$, the modified optimization problem is equivalent to the original optimization problem, and, thus, the two problems have the same computational complexity.

\section{Simulation Results}

Direct-sequence code-division multiple-access systems with the number of antenna elements ranging from three to eight 


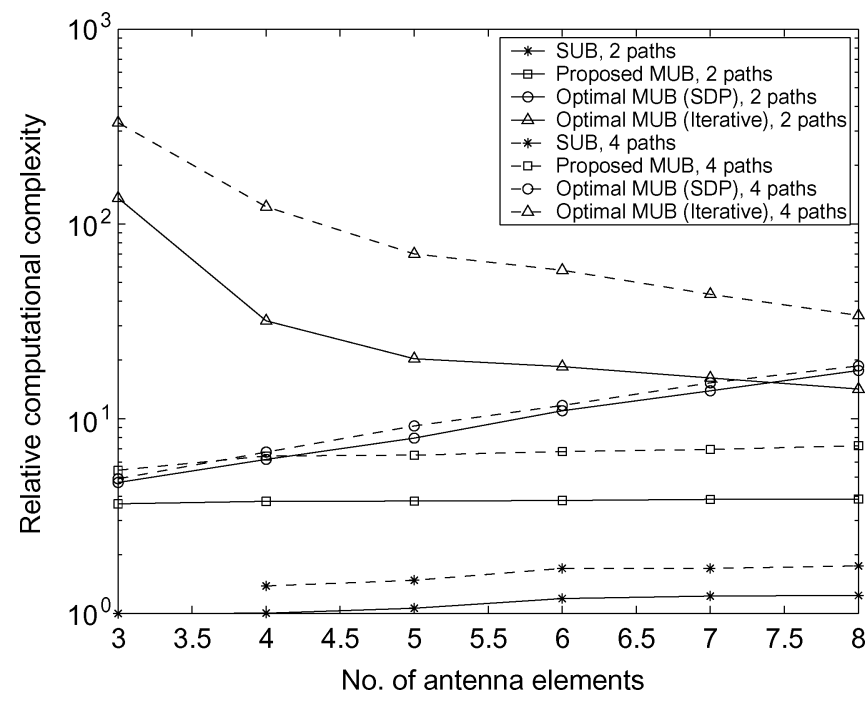

Fig. 2. Comparison of computational complexity.

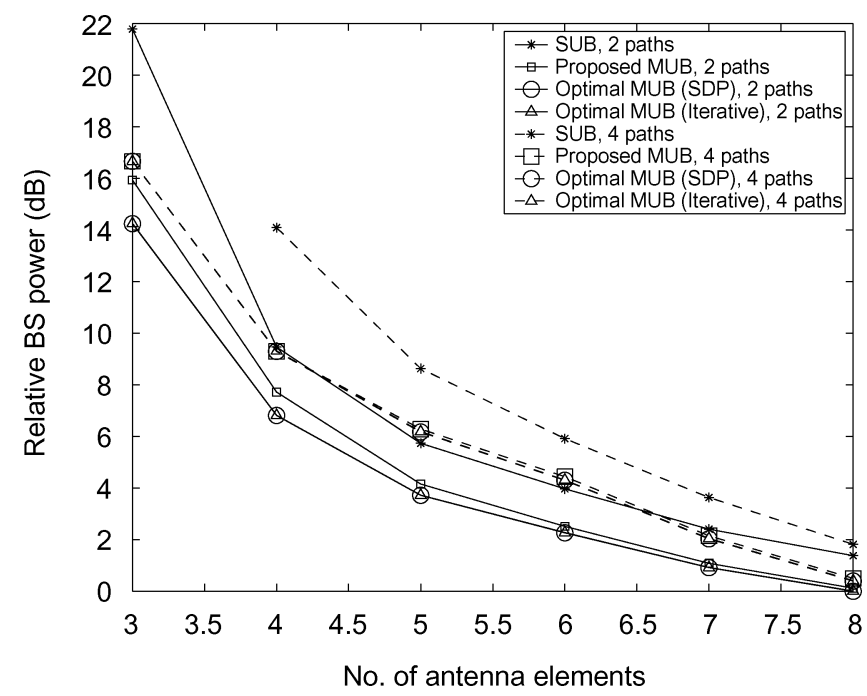

Fig. 3. Comparison of BS transmitted power.

in the BS array were simulated. The information bit-to-background power spectral density ratio $\left(E_{b} / N_{0}\right)$ of the received signal was set to $10 \mathrm{~dB}$ and $\gamma_{k}$ was set to $-10 \mathrm{~dB}$ for $k=$ $1,2, \ldots, K$. Twenty MSs and an angle of path spread of $\pi / 3$ for each MS were assumed. The spacing of the antenna array was set to $d=\lambda / 2$. The performance measures in the simulation were the total power transmitted from the BS and the computational complexity in terms of CPU time averaged over 1000 runs. In each run, the SUB [6], the proposed MUB, the optimal MUB using SDP [11], and the optimal MUB exploiting the duality between the uplink and the downlink [6] were simulated. As can be seen in Fig. 2, the computational complexity of the proposed MUB algorithm is significantly reduced relative to that of the optimal MUB. The MUB is insensitive to the increase of antenna elements since the number of variables in the SDP depends only on the downlink channels whereas the number of variables and, in turn, the computational complexity of the optimal MUB using SDP increases rapidly with the number of antenna elements. This confirms the discussion in Section III-B. It can also be observed that, when the number of antenna elements is small, the optimal MUB algorithm that exploits the duality between the uplink and the downlink requires high computational complexity due to its low rate of convergence in the case of a stringent SINR specification. The relative power transmitted from the BS versus the number of antenna elements is shown in Fig. 3. It can be observed that the total transmitted power using the proposed MUB algorithm approaches that achieved by the optimal MUB.

\section{CONCLUSION}

A new downlink MUB algorithm for mobile communications has been developed. In the new algorithm, the weight vectors of different MSs are jointly optimized in their corresponding subspaces instead of searching in the entire parameter space. Using the proposed algorithm, the number of variables considered in the SDP problem is reduced and its computational complexity is significantly reduced relative to that of the optimal MUB algorithms. In addition, the proposed algorithm leads to solutions that satisfy the SINR specification at each MS and at a total BS transmitted power which is close to the optimal one.

\section{REFERENCES}

[1] A. Yener, R. D. Yates, and S. Ulukus, "Interference management for CDMA systems through power control, multiuser detection, and beamforming," IEEE Trans. Commun., vol. 49, no. 7, pp. 1227-1239, Jul. 2001.

[2] R. Stridh, M. Bengtsson, and B. Ottersten, "System evaluation of optimal downlink beamforming in wireless communication," in Proc. IEEE Veh. Technol. Conf., 2001, vol. 5, pp. 1441-1445.

[3] P. Zetterberg and B. Ottersten, "The spectrum efficiency of a base station antenna array system for spatially selective transmission," IEEE Trans. Veh. Technol., vol. 44, no. 8, pp. 651-660, Aug. 1995.

[4] H. Asakura and T. Matsumoto, "Cooperative signal reception and down-link beam forming in cellular mobile communications," IEEE Trans. Veh. Technol., vol. 48, no. 3, pp. 333-341, Mar. 1999.

[5] J. Goldbery and J. R. Fonollosa, "Downlink beamforming for cellular mobile communications," in Proc. IEEE Veh. Technol. Conf., 1997, vol. 2, pp. 632-636.

[6] F. Rashid-Farrokhi, K. J. R. Liu, and L. Tassiulas, "Transmit beamforming and power control for cellular wireless systems," IEEE J. Sel. Areas Commun., vol. 16, no. 8, pp. 1437-1450, Oct. 1998.

[7] C. Farsakh and J. A. Nossek, "Channel allocation and downlink beamforming in an SDMA mobile radio system," in Proc. IEEE Int. Symp. Personal, Indoor Mobile Radio Commun. Conf., Sep. 1995, vol. 2, pp. 687-691.

[8] M. Schubert and H. Boche, "A unifying theory for uplink and downlink multi-user beamforming," in Proc. Int. Zurich Seminar Broadband Commun., Feb. 2002, pp. 27-1-27-6.

[9] —_ "Solvability of coupled downlink beamforming problems," in Proc. IEEE Global Telecommun. Conf., 2001, vol. 1, pp. 614-618.

[10] _ "Solution of the multiuser downlink beamforming problem with individual SINR constraints," IEEE Trans. Veh. Technol., vol. 53, no. 1, pp. 18-28, Jan. 2004.

[11] M. Bengtsson and B. Ottersten, "Optimal downlink beamforming using semidefinite optimization," in Proc. 37th Annu. Allerton Conf. Commun., Control, Computing, Sep. 1999, pp. 987-996.

[12] _ - "Downlink beamformer design using semidefinite optimization," in Proc. Radiovetenskap och Kommunikation, 1999, pp. 289-293.

[13] M. Bengtsson, "Jointly optimal downlink beamforming and base station assignment," in Proc. IEEE Int. Conf. Acoust., Speech, Signal Process., 2001, vol. 5, pp. 2961-2964.

[14] L. Vandenberghe and S. Boyd, "Semidefinite programming," SIAM Rev., vol. 38, pp. 49-95, Mar. 1996.

[15] D. Gerlach and A. Paulraj, "Adaptive transmitting antenna array with feedback," IEEE Signal Process. Lett., vol. 1, no. 10, pp. 150-152, Oct. 1994.

[16] N. Y. Wang, P. Agathoklis, and A. Antoniou, "A subspace multiuser beamforming algorithm for downlink of mobile communications," in Proc. IEEE Int. Symp. Circuits Syst., May 2004, vol. 3, pp. 381-384. 